Revue québécoise de psychologie

\title{
VISITE MÉDIATISÉE ET PSYCHOSE : UNE RÉFLEXION SUR LE CADRE
}

\section{MEDIATED VISIT AND PSYCHOSIS: A CLINICAL AND THEORETICAL FRAMEWORK}

\section{Khalid Boudarse}

Volume 39, numéro 1, 2018

URI : https://id.erudit.org/iderudit/1044848ar

DOI : https://doi.org/10.7202/1044848ar

Aller au sommaire du numéro

Éditeur(s)

Revue québécoise de psychologie

ISSN

2560-6530 (numérique)

Découvrir la revue

Citer cet article

Boudarse, K. (2018). VISITE MÉDIATISÉE ET PSYCHOSE : UNE RÉFLEXION SUR LE CADRE. Revue québécoise de psychologie, 39(1), 145-159.

https://doi.org/10.7202/1044848ar
Résumé de l'article

La visite médiatisée, un outil du champ médico-social en France, permet des contacts entre membres de la même famille en présence d'un professionnel. L'article vise à participer à la construction d'un cadre clinique et théorique relatif à cet outil en partant d'un dispositif de visite médiatisée tenu sur une durée de deux ans. Il a bénéficié à un père, souffrant d'une psychose non soignée, mais suffisamment compensée, et à son enfant de cinq ans victime de violences graves à l'âge de trois mois. Nous formulons l'hypothèse que le travail sur les frontières du cadre facilite le soutien de la personne psychotique en situation de visites médiatisées. 
VISITE MÉDIATISÉE ET PSYCHOSE : UNE RÉFLEXION SUR LE CADRE

MEDIATED VISIT AND PSYCHOSIS: A CLINICAL AND THEORETICAL FRAMEWORK

Khalid Boudarse ${ }^{1}$

Université de Rouen

Le présent article s'attache à décrire un dispositif de travail en protection de l'enfance, les visites médiatisées ${ }^{2}$, dans les situations où l'un des bénéficiaires présente une psychose. II a pour objectif de contribuer à l'élaboration d'un cadre théorique pouvant aider à penser cette pratique particulière. Pour ce faire, nous nous appuyons sur la présentation d'un cas clinique après avoir précisé les principaux termes théorico-clinique qui sont : visite médiatisée, protocole, cadre, psychose, frontières du moi et indifférenciation. Notre projet est de montrer comment l'approche topique, en terme de frontières du moi (Anzieu, 1985; Federn, 1979), et la conception des processus psychiques en œuvre dans la psychose et les pathologies limites peuvent doter les visites médiatisées du cadre dont elles sont dépourvues aujourd'hui. Nous terminerons l'article par une synthèse résumant nos propositions tant au niveau empirique qu'au niveau théorique.

\section{CADRE THÉORICO-CLINIQUE}

La visite médiatisée (VM), dispositif clinique, s'exerce en France principalement dans le champ de la protection de l'enfance. Elle procède d'une reconnaissance de l'intérêt d'une mise en contact entre personnes dans un espace institutionnel désigné par un magistrat en dépit des risques qu'une telle démarche comporte (séduction de l'enfant, exposition à la non-continuité, à la pathologie psychiatrique de l'autre, risque de passage à l'acte...). La visite médiatisée est ordonnée par le juge des affaires familiales et le juge des enfants. Elle est à distinguer des autres formes de rencontres en présence d'un tiers professionnel qui sont des dispositifs plus souples et pouvant être mis en place par les services de protection de l'enfance, par exemple sans passer nécessairement par une prescription judiciaire.

Contrairement à l'entretien ou à l'observation, les visites médiatisées n'ont pas de cadre théorique reconnu (Sellenet, 2011), ce qui peut amener

1. Adresse de correspondance : Université de Rouen, 25, rue du contrat social 76000 Rouen, France. Téléphone: $\left.\begin{array}{lllllll}00 & 33\end{array}\right) \quad 2 \quad 35 \quad 71 \quad 76 \quad 82 . \quad$ Courriel : kboudarse.elan@wanadoo.fr

2. Au Québec ce dispositif est nommé « visites supervisées ». Les lois de 2007, 2016 et 2017 relatives à la protection de l'enfance en France utilisent les mots « rencontres en présence d'un tiers ". 
des pratiques très différentes d'un service à un autre, voire d'un professionnel à un autre. Se pose alors la question du cadre pouvant aider à mener à bien non seulement le déroulement des contacts physiques, par exemple, mais aussi l'élaboration des processus psychiques qui s'engagent durant les visites entre l'ensemble des protagonistes : parents, enfants et professionnels. Notre question est donc: comment penser le cadre de ces visites? Cette question prend un relief singulier lorsqu'un bénéficiaire ou plus des VM présente une pathologie de l'ordre de la psychose.

Nous tenterons d'apporter quelques réponses à ces interrogations en ayant comme hypothèse de travail l'idée suivante : l'aspect contraint et souvent contraignant des VM oblige le clinicien à «bien » cerner les frontières de son cadre, à le signifier fréquemment, à le faire respecter et parfois à le faire évoluer. Cette conception du travail avec les limites du cadre nous paraît décisive pour mener à bien les VM, ceci d'autant plus important que l'un des bénéficiaires présente une altération des limites du moi, au sens de Paul Federn (Federn, 1979), tel que c'est le cas dans la situation clinique d'où nous partons. La limite est par ailleurs éthique : devons-nous renouer une relation lorsqu'un parent a largement montré sa nocivité par rapport à son enfant? Comment peut-on penser des contraintes qui s'opposent (Morin, 2004), comme quand l'intérêt de l'un des parents s'oppose à celui de l'autre parent, piégeant de fait le vécu de leur enfant?

S'inspirant des travaux de Maurice Berger (Berger, 2003, 2011), nous avons mis en place le protocole suivant : du point de vue organisationnel, un binôme Éducatrice-Psychologue ${ }^{3}$ rencontre à trois reprises le parent demandeur avant de se prononcer sur la mise en place des visites, le demandeur en est informé dès le premier entretien. Lorsque le binôme s'engage dans la réalisation des visites, il centre son travail sur « ce qui se passe ici et maintenant, durant la visite ", tout ce qui concerne la coordination avec l'extérieur (familles et partenaires) étant assuré par une autre éducatrice qui ne participe pas aux VM, mais qui échange régulièrement avec le binôme psychologue-éducatrice. Les visites sont mensuelles et se déroulent sur une heure, à l'exclusion des deux premières que l'on peut raccourcir quand c'est nécessaire afin de prévenir une tension trop forte, une agitation ou toute autre manifestation que les professionnels ressentent comme éprouvante pour les protagonistes. Un échange post-séance est pratiqué systématiquement par le binôme Éducateur-Psychologue. Un bilan est organisé toutes les trois séances.

3. Très souvent, en protection de l'enfance, les VM sont menées par des éducateurs spécialisés. La présence du psychologue dans la situation clinique que nous abordons ici est rendue nécessaire du fait de la pathologie mentale, une psychose diagnostiquée par le service de psychiatrie, et non soignée du demandeur, le père. 
Son objet concerne le respect des règles du cadre (ponctualité, régularité, non-intrusion, sobriété...), la réflexion sur les ressentis du bénéficiaire lors des visites précédentes, tant par rapport à l'enfant que vis-à-vis du cadre posé et à la restitution du point de vue des professionnels sur ces mêmes visites. Enfin, les audiences du Magistrat avec la famille et les deux éducatrices ponctuent l'évolution du processus. Notons que l'explicitation et la discussion du protocole, cadre et règles ont lieu pendant les trois premières rencontres avec le demandeur et que ses aspects peuvent être débattus, repris et évoluer lors des rencontres bilans.

Le cadre est un non-processus qui permet le déploiement d'un processus (Bleger, 1966). Ses règles principales dans les VM sont: le binôme de professionnels, la régularité, la ponctualité, la protection des frontières physiques et psychiques de chacun et la facilitation des interactions entre protagonistes. Lorsqu'il y a psychose, le travail sur les frontières, celles du cadre comme celles des protagonistes, et le souci de leur protection nous semble constituer l'élément déterminant dans l'accompagnement. Précisons que l'objet principal de ce cadre est d'élaborer à partir des interrelations telles qu'elles émergent en séance et que l'intervenant fait partie de cette dynamique parce qu'il est partie du néo-groupe (Granjon, 2006, 2016) qu'il forme avec les autres protagonistes présents.

Pour Berger (Berger, 2003, 2011) l'objectif des VM est la protection de l'enfant, non pas le soin des liens parents-enfant. II est question de favoriser l'évolution psychique de l'enfant en lui permettant, par des rencontres protégées de la toxicité parentale, de se différencier de ses ascendants. II s'agit de différencier ce qui lui appartient de ce qui provient de ses parents, délimiter un interne et un externe, discriminer ses émotions de celles d'autrui. Soutenir l'autonomie psychique de l'enfant passe alors par un dispositif "sécure » facilitant à l'enfant la confrontation avec «la réalité » des siens. La finalité ultime de la démarche selon cet auteur est de déculpabiliser l'enfant et de lui permettre de dépasser l'idéalisation de ses parents. A contrario, l'indifférenciation qui peut être suscitée et maintenue par le(s) parent(s) expose la maturation de l'enfant et son autonomie psychique. Pour Racamier (Racamier, 1993, 1995) l'indifférenciation est le produit de la dynamique incestuelle et de la perversion narcissique qui sont repérables dans le fonctionnement psychotique à l'échelle individuelle et familiale. Cette perversion est une perversion d'une dynamique ordinaire, celle de la séduction narcissique qui est à la base des toutes premières relations mère-bébé. Elle est une illusion dans le sens de Winnicott (Winnicott, 1971) qui perdure de façon pathologique, abolissant les frontières entre soi et non-soi. Sur le plan des relations interpersonnelles, l'incestuel manie la paradoxalité, la confusion générationnelle et démantèle l'espace intermédiaire (Boudarse, 2016). 
Dans le prolongement des thèses de Racamier, Caillot (Caillot, 2015) montre comment les familles à transactions psychotiques occupent une position narcissique paradoxale, oscillant entre un pôle narcissique organisé autour du fantasme de la famille idéale et un pôle antinarcissique caractérisé par le fantasme de délabrement familial. Enfin, au niveau métapsychologique, Roussillon saisit la paradoxalité qui est en œuvre dans le fonctionnement psychotique dans le vécu transférentiel: contrairement au transfert par déplacement caractéristique de la névrose, le transfert par retournement (Roussillon, 1991) désigne ce que le patient psychotique fait vivre au psychanalyste à défaut de pouvoir le vivre luimême. Ces repères théorico-cliniques accordant une place importante à la topique du moi et aux processus psychotiques nous paraissent nécessaires pour construire un cadre théorique congruent avec la pratique des VM lorsque domine un fonctionnement psychotique chez l'un des bénéficiaires. La situation clinique que nous présentons maintenant tente de montrer la pertinence de leur maniement.

\section{CLINIQUE}

Monsieur $\mathrm{Michel}^{4}$, une trentaine d'années, séparé de la mère de sa fille Françoise âgée de cinq ans, partage sa vie avec Madame A, de quelques années plus jeune que lui, de qui il attend une fille. Les VM concernent la dyade père-fille. La mère de Françoise, Andrée, la trentaine, est mariée avec un homme du même âge qu'elle. Le couple a une fille de trois ans et attend une deuxième fille. Monsieur Michel présente une psychose, diagnostiquée par le service de psychiatrie, plus ou moins compensée et non soignée. Madame Andrée est déficiente intellectuellement et manifeste régulièrement des accès hypomaniaques.

Les parents de Françoise se sont séparés à l'incarcération de Monsieur Michel à la suite des maltraitances graves, dont il a été reconnu coupable par la justice, sur Françoise alors âgée de moins de trois mois. Françoise a été placée puis, quatre ans plus tard, a intégré une " nouvelle » famille maternelle puisque sa mère partage sa vie avec un autre homme avec qui elle a un enfant. La fillette a cinq ans lorsque le juge des enfants ordonne des visites médiatisées avec son père. C'est dans ce contexte que nous avons accompagné la dyade père-fille, puis la famille.

4. Pour des raisons de confidentialité, tous les noms propres sont modifiés. 


\section{LES PREMIĖRES SÉANCES}

La fragilité des limites se manifeste dès l'entrée en contact avec Monsieur Michel. II est convaincu de la mauvaise image que nous avons de lui, persuadé que son ex-compagne nous aurait parlé de lui en le dénigrant, elle était du reste susceptible d'ouvrir à tout moment la porte de la pièce où nous nous rencontrions pour nous dire des choses négatives sur lui. Il angoisse à l'idée que l'on puisse raconter ses paroles à autrui. Ici, notre appui sur des éléments du protocole et du cadre (le binôme psychologue-éducatrice n'a aucun lien particulier avec la mère de l'enfant et l'éducatrice de l'Action Éducative en Milieu Ouvert [AEMO] ${ }^{5}$ n'a aucun contact avec Monsieur Michel) a autorisé une élaboration minimale de ces angoisses, du moins leur contenance temporaire.

Projectif et en proie à des angoisses paranoïdes, Monsieur Michel redoute qu'en nous parlant nous utilisions ses paroles contre lui. II s'agit d'une angoisse se manifestant, au début de l'accompagnement, de façon permanente à chaque visite et à laquelle nous réagissons par une métaphore: « rien ne franchira cette porte de notre part sans que vous n'en soyez averti ». Pour Serge Lebovici (Lebovici, 2002), la métaphore est un efficient outil thérapeutique. Le passage de l'objet concret " porte » à sa métaphorisation en termes de clôture interne a constitué une avancée considérable dans le travail mené avec Monsieur Michel.

De ce point de vue, investissement des limites et contenance vont de pair. En effet, l'attaque de nos liens de travail par des sentiments de persécution et d'empiétement menaçait en permanence la continuité du travail. C'est comme si l'angoisse de persécution et de perforation des enveloppes internes de Monsieur Michel avait été projetée sur notre cadre, cet autre intérieur que nous tenons à protéger. Ainsi, l'angoisse d'être contaminé par l'extérieur, vécue par ce père, est "endiguée » en partie par un étayage sur l'une des règles prescrites et respectées.

Tester à plusieurs reprises les limites et la consistance du cadre fut nécessaire pendant plusieurs mois à $\mathrm{M}$. Michel. Des demandes sollicitant notre intervention directe, les motivations rationnelles ne manquaient pas, auprès de la mère de Françoise en étaient l'exemple. Notre refus ferme et poli trouve son étayage sur le cadre posé (nous intervenons sur ce qui se passe ici et maintenant, à l'abri de tout autre regard ou contact avec les autres membres de la famille) et a le souci de renvoyer un sens quant à la consistance des frontières. Ainsi, moins les frontières du sujet sont investies plus la clarté des frontières du cadre paraît nécessaire.

5. L'AEMO est une assistance éducative judiciaire ordonnée par le juge des enfants. 


\section{LES PREMIERS CONTACTS PÈRE-FILLE}

$\mathrm{Au}$ tout début de la première séance, en présence du binôme psychologue-éducatrice et du père, Françoise âgée de cinq ans, assise à une petite table, jette un regard en direction de ce dernier qui lui dit bonjour et demande un "bisou ${ }^{6}$. Elle se détourne de lui et répond par un «non! » qu'elle lui oppose d'emblée et qu'elle maintiendra pendant longtemps relativement à toute sollicitation venant de la part de son ascendant. Ce dernier garde une distance physique avec sa fille et ne cherche pas à la toucher physiquement. Françoise joint le geste (des coups de pied en direction de son père, mais qui n'atteignent pas ce dernier) au mot « non! » Monsieur répond par un « si! » qui perdurera, lui aussi, longtemps. Un embryon de jeu, d'un espace intermédiaire, est bien là. Dans un troisième temps, Françoise se réfugie sous le siège de l'éducatrice assise dans un coin de la salle. Elle est alors comme quadrillée, protégée, par les pieds de la chaise et les jambes de l'éducatrice. Au " non », accompagné du geste de pied, s'ajoute ainsi une nouvelle barrière " protectrice » de l'enfant. Quelques instants plus tard, la fillette rencontre de la part de l'éducatrice un autre «non! », un interdit de donner des coups de pieds. Interdit protecteur du père et prescripteur d'une limite à l'agressivité de l'enfant. Lors d'une autre séance, un " non » spontané a été adressé par la même professionnelle au père afin d'assurer ce qui a été ressenti par elle dans le vif des échanges comme un besoin de barrière protectrice de la fillette. L'angoisse de l'intrusion et de la décontenance que cela pourrait susciter appelle le besoin d'ériger symboliquement et même physiquement une barrière de contact. Ce ressenti est au cœur d'un souci consistant à protéger les frontières de chacun. Ainsi, le respect de la barrière de contact manifesté, défendu même par l'enfant, par nous et par le père est un indicateur déterminant dans la poursuite des visites. Notons au passage la bipartition "spontanée» des sensibilités: l'éducatrice est plus proche émotionnellement de l'enfant, le psychologue l'est du père.

À la séance suivante, Françoise arrive au rendez-vous en retard et en pleurs. L'enfant est fatiguée, angoissée. Elle refuse tout contact visuel avec son père. Néanmoins, elle lui adresse régulièrement un regard discret. Les principaux échanges verbaux, menés par le psychologue, se dérouleront entre les professionnels et Monsieur Michel qui évoque sa filiation. II fait des recherches généalogiques sur sa famille depuis plusieurs années. Ainsi, pendant une bonne partie de cette séance, le père évoque ses ancêtres. Françoise écoute, nichée dans les bras de l'éducatrice. Lorsque celle-ci se relâche, Françoise lui prend les bras et les réajuste autour d'elle. Puis la fillette tonna dans un élan de colère et de tristesse que son père est celui qui est en bas dans la salle d'attente, à

6. Le mot est du père. 
savoir son beau-père. En effet, avant les visites médiatisées, Françoise avait un père en la personne du compagnon de sa mère. Aujourd'hui, elle en a un deuxième, "flanqué " d'une éducatrice et d'un psychologue. Le père géniteur paraît ainsi pour la fillette comme un étranger qui angoisse, cible d'attaques et source de curiosité. Nous reconnaissons dans ce vécu ce que, à la suite des travaux de René Spitz (Spitz, 1965), décrit Margaret Mahler (Mahler, 1975) de l'enfant du huitième mois en présence de l'étranger dont le prototype est justement le père : l'angoisse et la curiosité se manifestent ensemble. Cette réaction de Françoise signe une évolution favorable de la relation à son père et plus globalement un réaménagement interne de son économie psychique.

\section{ÉVOLUTION DES INTERACTIONS PĖRE-FILLE. ÉVOLUTION DU CADRE}

Six séances sont passées. La rigidité de l'opposition du « Non » et du " $\mathrm{Si}$ » s'est transformée en un jeu souple entre père et fille, tout comme la qualité du rapproché physique entre eux. Entre-temps, le regard discret et quasi "volé » de Françoise en direction du regard de son père a pu évoluer vers des échanges prolongés. Faire des jeux de construction (la fillette ne figure rien encore lorsqu'elle dessine) devient possible pour la dyade. La relation s'apaise donc et les interactions s'harmonisent... à condition que Françoise garde la maîtrise, pilote et que le père accepte de s'accorder au rythme de sa fille en adoptant une certaine passivité. L'attitude paternelle a facilité l'assouplissement des défenses de Françoise.

Monsieur Michel continue à s'étayer sur nous. II reprend certaines de nos interrogations à son compte, notre manière de nous intéresser aux jeux, dessins et certaines conduites de son enfant. II semble faire sien, internaliser donc ce qu'il retient de nous. Notons enfin qu'à cette phase des visites, en séance, Monsieur Michel n'est plus en proie à des idées délirantes, il n'est plus intrusif et ses interactions avec sa fille sont de plus en plus harmonieuses. De son côté, Françoise se montre moins rigide, car moins angoissée. L'accordage affectif et comportemental de la dyade est observable en séance. Au regard de cette évolution, nous décidons de faire évoluer le cadre : nous passons des VM à l'entretien familial.

\section{LES ENTRETIENS FAMILIAUX}

Les entretiens familiaux intègrent, en plus de la dyade concernée, la compagne de Monsieur Michel et leur bébé âgé d'environ huit mois. Ces rencontres ont lieu une fois par mois et se déroulent sur une heure. En dehors de ces entretiens, le magistrat des enfants a accordé un « droit de visite » d'une demi-journée par quinzaine à la famille paternelle, la présence de la compagne de Monsieur Michel étant exigée. 
L'amorce des entretiens familiaux se révèle difficile: les adultes évoquent peu de choses à propos de leur relation à Françoise; un sentiment d'ennui résonne de part et d'autre chez les professionnels et les adultes; et la fillette s'agite et retrouve des défenses que l'on croyait assouplies (repli, rigidité des conduites, contrôle obsessionnel, nervosité...). Malgré la difficulté et le doute qui s'est emparé de nous quant à la pertinence du nouveau dispositif, le cadre est maintenu et est respecté par la famille.

Un tournant se produit lorsque le père et sa compagne évoquent des éléments semblables de leurs histoires familiales respectives qui croisent leur intérêt pour la généalogie. Des événements traumatiques ont jalonné I'histoire familiale de Monsieur et dont il a une certaine idée, sites spécialisés sur internet aidant. Du côté de la compagne, des événements traumatiques, des meurtres, sont, selon elle, « rapportés, racontés " dans la famille, mais leur contexte et les motivations de leurs acteurs sont obscures et suscitent chez elle un désir de savoir. Désir contre lequel Monsieur avertit parce que c'est "douloureux », précise-t-il. II le sait, ajoute-t-il, pour l'avoir vécu. Empathie et identification s'expriment ainsi. Entre temps, Françoise s'apaise, échange, partage son jeu avec sa sœur et commence à dessiner des figures reconnaissables et à propos desquelles elle peut "raconter une histoire ». S'étayant sur nous, Monsieur s'intéresse aux productions graphiques et aux jeux de sa fille. Les dernières séances sont paisibles, la réciprocité des échanges perdure et l'ambiance affective dominante est positive. Nous évoquons la fin de nos rencontres et signifions aux protagonistes notre permanence, ils peuvent nous solliciter plus tard en cas de besoin. Le Juge des Enfants accepte et valide en audience en présence de la famille et des éducatrices notre proposition de la fin des entretiens familiaux et de l'accompagnement éducatif. Désormais, les parents s'organisent pour les droits de visite et d'hébergement.

\section{DISCUSSION}

Que retenons-nous du cadre élaboré pour cet accompagnement? Comment ce cadre peut-il accueillir et aider des protagonistes attaqués narcissiquement et dont les frontières du moi ne sont pas suffisamment investies?

Les projections et associations délirantes de Monsieur Michel, nous l'avons vu, ont d'abord pris comme support les frontières de notre cadre. Endiguer les effets de ce fonctionnement du côté de Monsieur Michel a comme corollaire la contenance de l'excitation et de l'agressivité du côté de Françoise. Ainsi, l'angoisse d'intrusion, d'empiétement, semble former l'affect dominant dans l'espace psychique dyadique père-fille. Elle a 
constitué un courant de fond ayant traversé la majorité des séances. La fragilité narcissique de la dyade est telle qu'elle n'a pas manqué d'éprouver émotionnellement plus d'une fois le binôme éducatricepsychologue et a testé la consistance de leurs gestes et paroles (angoisses massives du père et risques de passage à l'acte). Notons enfin que l'effort de maintenir un cadre clairement défini pour les VM a permis au père de Françoise d'identifier progressivement un espace, lieu conteneur d'interactions comportementales, affectives et imaginaires, et enveloppe contenant (Kaës, 1993) et visant l'élaboration de ce qui est déposé ici et maintenant.

Différenciation des espaces et prescriptions des limites se retrouvent par ailleurs et de façon plus générale dans le protocole et cadre adoptés, dans le non-processus (Bleger, 1966). Par exemple, l'intervention d'un binôme de professionnels centrant son intervention sur les visites uniquement paraît nécessaire, laissant à un référent, un troisième professionnel donc, la charge d'organiser ou d'accompagner la famille de façon générale et d'assurer le travail de liaison avec les autres partenaires institutionnels. Les frontières du cadre se trouvent ainsi prescrites pour l'ensemble des protagonistes formant le néo-groupe. Le sens de la démarche est que le cadre s'impose à tous. Par ailleurs le centrage du travail se traduit par le souci de le circonscrire à ce qui se déroule " ici et maintenant » dans l'espace des visites, limitant l'intervention des professionnels sur les interactions auxquelles ils participent. Centrer l'élaboration sur les interactions avec ses différents niveaux, comportemental, affectif et imaginaire, convoquent des concepts élaborés par les études relatives aux interactions précoces parents-bébé et plus généralement les travaux sur l'intersubjectivité (Ciccone, 2011; Lebovici, 2002). Ces concepts s'articulent avec ceux relatifs à l'approche de la psychose et des pathologies limites (Anzieu, 1985; Caillot, 2015; Federn, 1979; Fonagy, 2016; Racamier, 1995; Roussillon, 1991)

Soulignons un point commun entre le cadre et le Moi : le cadre des VM est défini par deux frontières principales, l'une tournée vers l'extérieure (assurée par l'éducatrice de l'AEMO) et l'autre vers l'intérieur (animée par le binôme), tout comme le Moi (Federn, 1979) qui possède une frontière externe (le moi par rapport au monde extérieur) et une interne (entre l'inconscient et le conscient). Aussi, lorsque les frontières psychiques du sujet sont menacées ou mal établies, leur étayage sur les frontières du cadre soutient leur réinvestissement. II nous semble que le souci de fixer des frontières de plus en plus investies au fil des séances était une démarche particulièrement aidante pour Monsieur Michel et sa fille.

La modification du cadre (passage des VM aux entretiens familiaux) montre comment le changement génère de fortes angoisses et provoque 
une rigidité des défenses. Ces dernières sollicitent émotionnellement de façon forte le néo-groupe. Une dynamique similaire est rapportée dans le cas Carine (Berger, 2002), enfant placée dès la naissance et suivie pendant plus de dix ans dans le cadre de VM avec sa mère. L'intérêt du travail en binôme dans le cadre d'une équipe stable y est, par ailleurs, clairement démontré. Le cas Carine illustre les repères théoriques et cliniques proposés par l'auteur (Berger, 2011, 2002) dont deux sont essentiels : permettre des contacts dans un cadre sécure et soutenir la différenciation parent-enfant en étayant la maturation psychique de l'enfant. Ce second point est caractéristique de l'approche de l'auteur qui insiste sur l'intérêt d'accompagner le changement des représentations que l'enfant s'est construit de ses parents. En effet, en mesurant les défaillances et les limites de ses parents, en renonçant à leur idéalisation, l'enfant vivrait son placement et la limitation de ses contacts avec les siens avec moins de culpabilité. Selon le même auteur, le travail gagnerait à être centré sur le potentiel évolutif de l'enfant, non sur celui du parent psychotique. En dépit de l'indéniable intérêt clinique de cette position, il nous semble qu'elle mérite d'être nuancée. II y a des liens parents-enfant qui évoluent favorablement, le cas clinique que nous avons exposé le montre bien.

Les processus interpersonnels et transférentiels demandent une élaboration au cours des séances et pendant les post-séances en abordant doute, hésitation, raideur, inquiétude... vécus par les professionnels. Les échanges après les séances n'ont pas comme intérêt pour ces derniers la seule recherche d'un minimum d'accordage, mais une occasion de mettre en mots la sensibilité de chacun telle qu'elle s'est montrée en séance et la mise à jour de ce que cette sensibilité aurait suscité chez les autres protagonistes. La perspective intègre donc les attitudes internes de chacun, leurs résonances ainsi que leur mise en commun, soit les liens transfert/contre-transfert/intertransfert. Le modèle théorico-clinique le plus proche de cette démarche est celui de la psychothérapie familiale psychanalytique (Caillot, 2015; Eiguer, 1989; Kaës, 1993; Lemaire, 2007). Ce modèle est caractérisé par la prise en compte de l'activité psychique consciente et inconsciente de chaque protagoniste de la relation et de l'interfantasmatisation que cette activité produit en situation du néo-groupe formé par la famille et les professionnels. La centration de l'élaboration sur les liens intersubjectifs principalement, et non sur la subjectivité de chaque sujet, suscite l'expression de la part indifférenciée qui demeure en chacun et qui s'exprime dans l'ici et maintenant des rencontres par les mots, les gestes, les mimiques, les postures physiques et l'utilisation des objets médiateurs (récit de rêves, psychodrame, dessin, génogramme...). Soutenir le travail de différenciation revient à créer les conditions de l'instauration d'un espace intermédiaire. Notons au passage que le souci d'accompagner les bénéficiaires dans l'appréhension des interactions auxquelles ils 
participent dans l'ici et maintenant évoque la psychothérapie basée sur la mentalisation que Fonagy et Bateman (Bateman et Fonagy, 2006; Fonagy, 2016) préconisent pour les soins des pathologies limites: soutenir dans le lien thérapeutique l'accès du patient à ses émotions et représentations ainsi qu'à celles des autres.

Insistons sur l'intérêt porté à la sensibilité des frontières qui prend des formes diverses. En effet, la limite protectrice de l'intégrité du moi de chacun est assurée du mieux possible par l'investissement des frontières du cadre et de ses règles, par le souci de centrer le travail sur ce qui se déroule en séance et par le renoncement au désir d'approfondir la " connaissance » des histoires individuelles. Précisons ce dernier point: échanger à propos de l'histoire de chacun est nécessaire pour soutenir la circulation psychique dans les dyades et les familles, mais il est nécessaire de protéger le cadre en ne cédant pas à la tentation d'écouter plus avant des récits pouvant décentrer le travail portant sur les interactions vers un abord concernant davantage le fonctionnement intrapsychique de chaque sujet ou le dévier vers des interactions " périphériques ». À titre d'exemple, soutenir le récit de Monsieur Michel relatif à sa filiation nous a semblé nécessaire pour maintenir un lien qui ne pouvait tolérer la proximité physique à un moment où l'enfant montrait son besoin de protection contre cette dernière, dans un premier temps; dans un second temps, le contenu de ce récit portant sur la filiation insérait Françoise dans une lignée. $A$ contrario et à titre d'exemple, répondre au désir de la nouvelle compagne de Monsieur d'aborder des aspects traumatiques de son histoire familiale débordait les limites du cadre. II en est de même lorsqu'elle nous interroge sur " l'aptitude » de Monsieur à être père. Par ailleurs, avoir comme repère l'idée de mettre une limite à son écoute facilite le passage du cadre rigide des visites médiatisées au cadre plus souple des entretiens familiaux.

En outre, et en règle générale, les visites médiatisées autorisent des contacts entre enfants et parents dans un cadre garanti par des professionnels. Cela suppose que ce contact recèle en lui des risques tels que le passage à l'acte agressif, l'intrusion... La dimension de l'acte et de l'action y est importante dans la mesure où le dispositif ne vise pas en premier lieu et de prime abord la production de la parole, comme c'est le cas pour l'entretien. Par exemple, le besoin de toucher, d'étreindre est souvent là avec son corollaire angoissant, la peur d'échouer, d'être rejeté ou de passer à l'acte. Dans ce sens, l'attention portée au vécu de l'enfant demeure primordiale (Sellenet, 2011). Par ailleurs, le désir de l'un peut être vécu comme une intrusion de la part de l'autre. Aussi est-il nécessaire de veiller à la mise en place d'un cadre sécure et facilitateur. Enfin, l'acte et l'action ne sont pas réductibles aux seuls passages à l'acte, le passage par l'acte n'étant pas toujours un court-circuit de la pensée. 
La capacité de jouer est un critère décisif ou du moins très important dans l'évolution positive des visites. Le jeu conçu comme un espace en mouvement, lieu de créativité animé par le chevauchement des frontières psychiques (Winnicott, 1971). C'est ce qui s'est dessiné sous forme embryonnaire dès la première rencontre. Le développement de cette capacité a suivi l'apaisement des angoisses dans la dyade. Son amorce a commencé d'abord par la reconnaissance et la protection des frontières fragiles de chaque protagoniste. Elle a traversé une crise lors du changement du cadre.

La reprise du jeu dans le nouveau cadre (entretiens familiaux) est passée par la difficile "acceptation" de la dispersion psychique de Françoise et des mécanismes obsessionnels réinvestis. Une alliance de travail bien établie a permis de " tenir » face à la destructivité de l'enfant. Selma Fraiberg écrit à propos de l'alliance : "Quel que soit le cas, c'est toujours le même principe. Une relation thérapeutique commence avec un "besoin" et "quelqu'un qui répond à ce besoin" », (Fraiberg, 1989, p. 47). La demande explicite de Monsieur Michel, à l'origine des visites et d'où découle le transfert initial, était de rencontrer sa fille. Après-coup, cette demande nous semble avoir été portée par une attente non formulée. Elle a trait aux soins à apporter à ses frontières internes à lui. Saisi par l'entremise du contre-transfert, cette attente s'est traduite, nous semble-t-il, par un souci permanent de porter soins aux frontières de notre cadre.

\section{POUR CONCLURE}

La VM s'amorce presque toujours dans un contexte contraint, par injonction judiciaire le plus souvent. Ce contexte est la conséquence d'un cadre légal ${ }^{7}$ et institutionnel visant la protection de l'enfant et le maintien de ses liens familiaux. Ce cadre forme le point de départ de toute VM et précise des limites qui s'imposent aux intervenants et aux bénéficiaires. La question de la limite est posée ainsi d'emblée et à tous. Elle est à la base de l'élaboration. Aussi, les repères théorico-cliniques gagnent à articuler l'approche topique en termes de frontières du Moi et l'analyse des processus psychotiques interpersonnels en termes d'incestualité, de paradoxalité et d'altération importante de l'espace intermédiaire. Quant au dispositif (protocole et cadre) construit avec les enfants et leurs familles, il a comme ossature quelques incontournables (Berger, 2003, 2011): d'un point de vue institutionnel, un protocole lisible pour les bénéficiaires, l'institution et les professionnels constitue le préalable à toute mise en contact entre bénéficiaires. En ce qui concerne la dynamique de travail, un cadre délimitant l'espace de son intervention est nécessaire tout en restant

7. Précisons une fois encore avec Charlet, magistrat, que les textes de loi ne mentionnent pas les termes "visites médiatisées ", mais utilisent les mots " rencontres en présence d'un tiers » (Charlet, 2015). Ce sont ces mêmes termes que la loi 2017 utilise. 
ouvert sur d'autres modalités d'accompagnement (consultation familiale ou de couple, suivi individuel) afin de ne pas couper les difficultés des protagonistes du contexte familial dans lequel elles s'expriment. Par ailleurs, la protection des frontières du cadre lui-même et de celles de chacun des bénéficiaires est au cœur du travail. Ceci est d'autant plus nécessaire lorsqu'il s'agit de psychose, car les frontières du cadre peuvent constituer un espace de projection pour celles, défaillantes, des protagonistes. Ainsi, la centration de l'élaboration sur les transactions interpersonnelles, le souci de prendre en considération les relations affectives (positives, négatives ou ambivalentes) se nouant dans le néogroupe constituent le matériel clinique et le moteur de l'accompagnement. Du côté des professionnels, la permanence et la prévisibilité comme la disponibilité interne et la facilitation des interactions sont les incontournables de leur cadre.

Sans les figer, soulignons enfin les indicateurs d'une évolution positive du processus de médiatisation : une acceptation minimale des règles par le(s) demandeur(s); une régression des risques d'intrusion de l'autre; une alliance de travail suffisamment assurée; et une capacité - ou du moins un potentiel - de jouer, au sens de Donald Winnicott (Winnicott, 1971). Les éléments favorisant une telle évolution d'un point de vue institutionnel sont un protocole et un cadre lisibles, une équipe de professionnels stable et un souci de penser en termes d'enveloppes de soins (complémentarité entre psychothérapie individuelle ou de groupe, VM, AEMO, psychothérapie familiale, placement...). Ces indications et cadre conceptuel que nous proposons méritent sans doute d'être plus affinés et complétés. Leur confrontation à une clinique où domine la perversion narcissique pourrait en être une occasion. Parce que cette perversion abolit les frontières entre les êtres que son étude éclairerait davantage un dispositif ayant comme souci premier de protéger contre l'empiétement et l'intrusion. II s'agit d'instaurer - du moins d'amorcer - un espace intermédiaire où le chevauchement des frontières psychiques est source de créativité, non de vampirisme psychique (Eiguer, 1989).

\section{RÉFÉRENCES}

Anzieu, D. (1985). Le Moi-peau. Paris, France : Dunod.

Bateman, A. et Fonagy, P. (2006). Mentalization-based treatment for borderline personality disorder : A practical guide. Oxford, Royaume-Uni : Oxford University Press.

Bateman, A. et Fonagy, P. (2015). Mentalisation et troubles de la personnalité limite : Guide pratique. Louvain, Belgique : De Boeck.

Berger, M. (2002). L'échec de la protection de l'enfance en danger, ou l'impossible changement. Devenir, 14(3), 197-238.

Berger, M. (2003). L'échec de la protection de l'enfance. Paris, France : Dunod.

Berger, M. (2011). Fondements théoriques et cliniques des visites médiatisées. Un dispositif en danger. Dans P. Gutton, A. Bagnulo et F. Jeanblanc (dir.), Les visites médiatisées dans le cadre de la protection de l'enfance (p. 61-83). Paris, France : Éditions GREUPP. 
Bleger, J. (1966). Psychanalyse du cadre psychanalytique. Dans R. Kaës (dr.), Crise, rupture et dépassement (p. 257-276). Paris, France : Dunod.

Boudarse, K. (2016). Narcissisme de couple et espace intermédiaire à l'épreuve de la psychose juvénile. Le Divan familial, 37(2), 141-152.

Caillot, J.-P. (2015). Le meurtriel, l'incestuel et le traumatique. Paris, France : Dunod.

Charlet, X. (2015). Les visites médiatisées selon l'ONED. Dans P. Gutton, A. Bagnulo et F. Jeanblanc (dir.), Les visites médiatisées dans le cadre de la protection de l'enfance (p. 11-20). Paris, France : Éditions GREUPP.

Ciccone, A. (2011). La psychanalyse à l'épreuve du bébé. Fondements de la position clinique. Paris, France : Dunod.

Eiguer, A. (1989). Le pervers narcissique et son complice. Pari, France : Dunod.

Federn, P. (1979). La psychologie du moi et les psychoses. Paris, France : PUF.

Fonagy, P. et Bateman, A. (2016). Préface. Dans M. Debbané (dir.), Mentaliser : De la théorie à la pratique clinique (p. 9-16). Louvain, Belgique : De Boeck.

Fraiberg, S. (1989). Fantômes dans la chambre d'enfants. Paris, France: Presses universitaires de France.

Fraiberg, S. (2012). Fantômes dans la chambre des enfants. Paris, France : PUF.

Granjon, E. (2007). Le néo-groupe, lieu d'élaboration du transgénérationnel. Le Divan familial, 18(1), 93-104.

Granjon, E. (2006). Le jeu en thérapie familiale psychanlytique. Le divan familial, 206(1), 4560.

Granjon, E. (2016). Jouer en thérapie familiale psychanalytique: objets bruts, objets de relation, objets médiateurs. Dialogue, 213(3), 25-40.

Kaës, R. (1993). Le Groupe et le sujet du groupe. Paris, France : Dunod.

Lebovici, S. (2002). Le Bébé, le Psychanalyste et la Métaphore. Paris, France : Odile Jacob.

Lemaire, J.G. (2007). Origine de l'idée fondatrice et évolution des thérapies familiales psychanalytiques. Dans J.-G. Lemaire (dir.), L'inconscient dans la famille (p. 1-30). Paris, France : Dunod.

Mahler, M. (1975). La naissance psychologique de l'être humain. Paris, France : PUF.

Morin, E. (2004). La méthode 6. Éthique. Paris, France : Seuil.

Racamier, P.-C. (1993). Cortège conceptuel. Paris, France : Apsygée.

Racamier, P.-C. (1995). L'inceste et l'incestuel. Paris, France : Éditions du collège.

Robert, P. (2007). La thérapie familiale psychanalytique: questions techniques. Dans J.-G. Lemaire (dir.), L'inconscient dans la famille (p. 57-107). Paris, France : Dunod.

Roussillon, R. (1991). Paradoxes et situations limites de la psychanalyse. Paris, France : PUF. Sellenet, C. (2011). Paroles d'enfants sur les "visites médiatisées » en protection de l'enfance. Dans P. Gutton, A. Bagnula et F. Jeanblanc (dir.), Les visites médiatisées dans le cadre de la protection de l'enfance (p.143-150). Paris, France: Éditions GREUPP.

Spitz, R. A. (1965). De la naissance à la parole. La première année de la vie. Paris, France : PUF.

Winnicott, D. W. (1971). Jeu et réalité : L'espace potentiel. Paris, France : Gallimard.

\section{RÉSUMÉ}

La visite médiatisée, un outil du champ médico-social en France, permet des contacts entre membres de la même famille en présence d'un professionnel. L'article vise à participer à la construction d'un cadre clinique et théorique relatif à cet outil en partant d'un dispositif de visite médiatisée tenu sur une durée de deux ans. Il a bénéficié à un père, souffrant d'une psychose non soignée, mais suffisamment compensée, et à son enfant de cinq ans victime de violences graves à l'âge de trois mois. Nous formulons l'hypothèse que le travail sur les frontières du cadre facilite le soutien de la personne psychotique en situation de visites médiatisées. 


\section{MOTS CLÉS}

visites médiatisées, visites supervisées, protection de l'enfance, maltraitance, psychose, pathologie des limites, interactions parent-enfant.

\section{ABSTRACT}

Supervised visits are a part of the tools used in French medico-social structures to allow contacts between different members of a family in the presence of a professional. This article intends to build a clinical and theoretical frame related to that specific tool. To do so, we will use a two-year clinical experience of supervised visits. It involved a father suffering from a psychosis that was not being treated but was adequately compensated, and his five-year-old child who had been the victim of aggravated violence inflicted since he was three months old. Our hypothesis states that working on the limits of the frame facilitates the support of the psychotic person in a context of supervised visits.

\section{KEY WORDS}

mediated visits, supervised visits, psychosis, framework, child welfare, violence, working in pairs, parents and children interactions. 